\title{
Surgical Treatment of Clinically Significant Reactive Hypoglycemia Nesidioblastosis, Post-Gastric Bypass
}

\author{
Ara Keshishian, MD, FACS, FASMBS ${ }^{*}$, Malgorzata Rajtar, $M D^{2}$ and Miguel Rosado, $M D^{3}$ \\ ${ }^{1}$ Department of Surgery, Huntington Memorial Hospital, USA \\ ${ }^{2}$ Research Associate, USA \\ ${ }^{3}$ Department of Physiology, Universidad De Guadalajara, Mexico
}

\begin{abstract}
Introduction: Gastric bypass (GB) used to be a standard surgical procedure performed for weight loss. Delayed complications following GB may outweigh the initial benefits in some patients. As manifested in some patients with clinical symptoms of hypoglycemia, Dumping Syndrome may, over time, progress to persistent Hyper-Insulinemic Hypoglycemia (HIH) and, in some cases, with Nesidioblastosis (NB) [1]. The current recommended surgical treatment includes $>95 \%$ pancreatectomy [2] which has been shown to cause irreversible diabetes in $90 \%$ of patients. We discuss fifteen patients who underwent gastric bypass revision to duodenal switch with a resolution of hypoglycemic symptoms.

Method: This is a retrospective analysis of prospectively collected data.

Results: Fifteen patients were seen and evaluated for clinically significant symptoms of hypoglycemia after the GB procedure. No insulinoma was discovered. Revision to Duodenal Switch (DS) was performed. Symptoms of HIH were reversed after surgery, and patients have remained $100 \%$ symptom-free post-operative follow-up.

Conclusion: In our experience, DS is a preferable operation for the correction of HIH. Duodenal switch shows greater efficacy with significantly fewer complications with tailored alimentary and standard channel lengths and should be considered before near-total pancreatectomy. Near-total pancreatectomy may be the last option for those who do not respond to the GB reversal.
\end{abstract}

\section{Keywords}

Gastric bypass, Duodenal switch, Revision, Hyper-insulinemic hypoglycemia, Neisidioblastosis, Pancreatectomy, Dumping syndrome

\section{Background}

Bariatric surgeries are the most effective intervention proven to result in significant and sustained weight loss and improvement or resolution of comorbidities associated with obesity, such as type II diabetes $[3,4]$. Different types of surgical weight loss procedures have varying degrees of safety and outcome profiles $[5,6]$. Roux-en-Y gastric bypass (GB) is a type of weight-loss surgical procedure for morbid obesity and its associated comorbidities. In GB, a small 2-3-ounce pouch is created just distal to the gastroesophageal junction and is connected to a Roux-en-Y limb created of the proximal small bowel. The stomach, pyloric valve, duodenum, and a varying segment of the jejunum are bypassed. There is nutritional and physiological sequala attributed to the exclusion of the pyloric valve from the digestive pathway [7]. Dumping syndrome and $\mathrm{HIH}$ have been recognized as a long-term complication of GB in a subset of patients $[8,9]$. In small series, near-total pancreatectomy has been recommended to treat clinically significant hypoglycemia and NB after gastric bypass operation with varying outcome [10].

Duodenal switch procedure was first described in 1987 as a surgical intervention to treat primary bile reflux gastritis or decrease symptoms in post gastrectomy and gastroduodenostomy patients [11]. This procedure was later adapted as a bariatric surgical solution for the treatment of morbid obesity by increasing longitudinal gastrectomy to $75 \%$, reducing gastric capacity and acid production, and extending the Roux limb to induce fat malabsorption resulting in weight loss

*Corresponding author: Ara Keshishian, MD, FACS, FASMBS, Department of Surgery, Huntington Memorial Hostpial, USA

Accepted: March 13, 2021

Published online: March 15, 2021

Citation: Keshishian A, Rajtar M, Rosado M (2021) Surgical Treatment of Clinically Significant Reactive Hypoglycemia Nesidioblastosis, Post-Gastric Bypass. J Surgical Endocrinol 3(1):73-76 
Citation: Keshishian A, Rajtar M, Rosado M (2021) Surgical Treatment of Clinically Significant Reactive Hypoglycemia Nesidioblastosis, PostGastric Bypass. J Surgical Endocrinol 3(1):73-76

$[12,13]$. In our experience, $\mathrm{HIH}$ can be reversed with GB to (DS) or Sleeve Gastrectomy revision while avoiding the complication of $80 \%$ pancreatectomy as described [14].

\section{Pathophysiology of Hypoglycemia in Post- RNY-GB Patients}

Nearly $50 \%$ of patients who undergo GB will develop dumping syndrome [15-17]. The dumping syndrome phenomenon is a postprandial disorder of rapid emptying of stomach pouch contents into the small bowel characterized by tachycardia, diaphoresis, dizziness, fatigue, and weakness $[16,17]$. The symptoms of hypoglycemia as a part of dumping syndrome may present early or late [18]. NB may develop in a group of patients with progressively advanced dumping syndrome over time.

$\mathrm{HIH}$ (plasma glucose $<50-55 \mathrm{mg} / \mathrm{dl}$ ) is characterized by neuroglycopenia (inadequate glucose supply of the brain) and inappropriately elevated insulin concentrations [19-21]. Our understanding of this condition has evolved over the years. $\mathrm{HIH}$ is thought to result as an acquired phenomenon or from the pancreas' failure to decrease insulin secretion after GB surgery adaptively [22]. The pylorus controls the flow of contents of the stomach into the small bowel. With the GB procedure, the duodenum and pyloric valve are entirely excluded. The pyloric valve's exclusion results in bolus entry of food in the small bowel in an unregulated format. Rapid presentation of nutrients into the small intestine allows for superfluous absorption of carbohydrates, especially after a carbohydrate-rich meal, stimulating $L$ cells of the distal ileum. This occurrence of the dumping syndrome is a stimulus of Glucagon-Like Peptide One (GLP-1) release [23]. GLP-1 stimulates regeneration and expansion of pancreatic beta-cells by process of neo-genesis and proliferation [24]. Studies have demonstrated the proliferative and anti-apoptotic properties of GLP-1 in human and rodent beta-cells.

Laboratory studies of rats treated with GLP-1 resulted in two significant findings:

1. An increase in cell proliferation of the endocrine and exocrine pancreas and

2. A reduction of apoptotic cells within the islets and exocrine pancreas [25].

Increased beta-cell mass induces hypoglycemia after GB surgery. The hypoglycemia is due to beta-cell proliferation and overactivity (Nesidioblastosis) as a result of GLP-1 stimulation [26]. Without pyloric regulation, dumping occurs, stimulating GLP-1, increasing proliferation, overactivity of Beta cells, and inhibiting programmed cell death of pancreatic islets, leading to inappropriately increased insulin-secreting cell hypertrophy $[24,25]$. Postprandial decreased blood glu- cose levels may present from endogenous hyperinsulinemia from resulting abnormal islets of Langerhans due to Neisidioblastosis observed in patients who have undergone GB as a treatment for severe obesity.

\section{Diagnosis of Hyper-Insulinemic Hypoglycemia}

No definitive standardized method for the diagnosis of $\mathrm{HIH}$ has been outlined. In addition to hypoglycemia symptoms, confirmation of the disorder ideally requires concomitantly elevated insulin ( $>3 \mu \mathrm{U} / \mathrm{ml})$, a negative oral hypoglycemia agent screen, and elevated C-peptide (>0.6 ng/ml) [18]. Hypothetically, during spontaneous postprandial episodes of hypoglycemia-related to hyperinsulinemia, C-peptide levels may be obtained due to its half-life (30 minutes). This finding is not a confirmation of excessive beta-cell secretion; instead, it rules out exogenous insulin administration, where levels would be low or undetectable. Provocative testing such as oral glucose tolerance test and mixed meal studies are suggested to induce hypoglycemia and its symptoms $[27,28]$. In our opinion, symptoms and laboratory studies differentiate the two conditions of Nesidioblastosis and insulinomas significantly that the CT scan may not be of any added value (Table 1). Insulinomas, insulin-secreting tumors of the pancreas, may cause hypoglycemia, but not postprandially. Patients diagnosed with insulinoma have increased insulin production that stimulates hypoglycemia during fasting. Hallmark symptoms of insulinoma are tachycardia, dizziness, and diaphoresis when patients do not feed frequently. On the contrary, $\mathrm{HIH}$ induced after GB surgery presents with hypoglycemic symptoms after feeding. Fasting ameliorates patients' hypoglycemic symptoms [9]. This correlates directly with inappropriate insulin secretion from hypertrophied beta-cells due to GLP-1 stimulation as a result of the rapid emptying of gastric contents into the small bowel with increased carbohydrate absorption after feeding.

\section{Current Recommendations of Treatment}

$\mathrm{HIH}$ and Nesidioblastosis have been reported to resolve over time in some patients and neuroglycopenia may not be evident [24]. Majority of patients suffering from HIH respond to dietary modification, such as eating frequent, small, low-carbohydrate, high protein meals. Medications to decrease the absorption rate of carbohydrates and hormone antagonists such as Acarbose and somatostatin respectively have been empirically associated with symptom modification in select patients as an aid to nutritional changes. Patient compliance and its associated cost are significant factors for their limited use. Carbohydrate inhibitors (such as Acarbose) in patients with dumping syndrome and $\mathrm{HIH}$ who do not follow dietary guidelines is contraindicated as it can create significant gastrointestinal adverse effects [2]. For those patients with

Table 1: Fasting vs. Posprandial Parameters of Insulinoma vs. Nesidioblastosis [23].

\begin{tabular}{|l|l|l|l|l|}
\hline & Fasting Insulin & Postprandial Insulin & $\begin{array}{l}\text { Fasting Glucose 60-110 } \\
\mathrm{mg} / \mathrm{dl}\end{array}$ & $\begin{array}{l}1 \text { hour post prandial Glucose } \\
\Uparrow \sim 140 \mathrm{mg} / \mathrm{dl}\end{array}$ \\
\hline Insulinoma & $\Uparrow$ & $\Uparrow$ & $\Uparrow$ & $\Uparrow$ \\
\hline Nesidioblastosis & $\Uparrow$ & $\Uparrow \Uparrow \Uparrow$ & $\Uparrow$ & $\Uparrow \Uparrow$ \\
\hline
\end{tabular}


HIH severe enough not-responding to nutritional intervention or medication, surgery has been advocated. The surgical procedure recommended has been $80-95 \%$ distal pancreatectomy [2]. This procedure reduces the amount of inappropriately secreted insulin, reducing hypoglycemia episodes by removing the cells that produce and release the insulin - Beta cells. Retrospectively, near-total pancreatectomy has been shown to cause hyperglycemia as a complication [29]. Since gastric feeding reverses the hypoglycemia after gastric bypass, a non-pancreatic etiology is possible. As an irreversible surgical operation, distal pancreatectomy, patients who are affected are no longer able to produce adequate insulin, leading to hyperglycemia and the associated complications. It has been shown that resections greater than $90 \%$ frequently result in insulin dependency and irreversible diabetes [14].

\section{Materials and Methods}

This is a retrospective study of prospectively gathered data. Fifteen patients presented with dumping and postprandial hypoglycemia symptoms as their primary and significant chief complaints that were affecting their quality of life. Patients were assessed and carefully considered for revision surgery. All fifteen patients underwent revision from GB to DS. The duodenal switch was performed with a length of the alimentary and common channels measured as a percentage of the small bowel's measured total length. Common and Alimentary channels were measured to $10 \%$ and $40 \%$ of the total small bowel length, respectively [13].

\section{Results}

A total of 15 patients were identified for this study. These include patients seen in our clinic for revision of failed GB, with a primary complaint of clinically significant dumping, hypoglycemia unresponsive to conservative treatment, including behaviors and dietary changes; the eligibility requirement was symptoms consistent with hypoglycemia following GB. Of these patients, 12 were females, and 3 were males; the mean age was 42.53 years with a standard deviation of 7.07. The mean weight and BMl at which these patients had their DS was 287.93 lbs. and 49.63 respectively; the mean weight and BMI before surgery for revision to DS was $223.13 \mathrm{lbs}$. and 37.92 respectively, and two years after DS was $158.4 \mathrm{lbs}$. and 27.52 with a mean reduction of the total weight of $64.73 \mathrm{lbs}$. and 10.4 of BMI.

Abdominal Ultrasound and CT scan or both had been used as a diagnostic study in all patients to rule out insulinoma. The weight loss peak occurred at 16 months at $115 \mathrm{lbs}$ (58-173). All of these patients had reported dumping syndrome, which did not respond to conservative treatment. In all cases, their symptoms worsened over time, with significant psycho-social complications impacting the regular and routine function of their everyday life. Patients underwent physical examinations and routine laboratory tests. The patients were evaluated by a complete medical history and physical exam. Their records, which included comprehensive metabolic workup and radiologic studies, were reviewed. None of these patients presented with fasting hypoglycemia. All patients had normal fasting blood glucose levels with significant symptomatic postprandial hypoglycemia diagnostic of Nesidioblastosis.
There was no radiologic evidence of insulinoma. They all had imaging studies, including CT scans and US. Six of the fifteen patients (all females) had a temporary gastrostomy tube inserted to improve their nutritional status before revision the GB to the DS operation. Their symptoms were absent with the feedings via the gastrostomy tube and present with oral intake. Placing a feeding tube in the remnant stomach allowed incorporating the previously omitted pyloric valve and regulated nutrient presentation to the small intestine.

Duodenal switch procedure, as mentioned previously, returns a function of the remnant stomach, pyloric valve, and duodenum and lengthening of the alimentary limb. By reinstating pyloric valve function, control of nutritional content entering into the small intestine ameliorated dumping syndrome, $\mathrm{HIH}$ and NB.

Patients following DS had complete resolution of $\mathrm{HIH}$ symptoms that were interfering with their quality of life. At the time of following, on average three years post-op, they all continue to be free of dumping syndrome symptoms and $\mathrm{HIH}$. DS has shown to carry no secondary risk of pancreatic dysfunction and production of insulin-dependent diabetes, as seen in $90 \%$ of patients who undergo pancreatectomy for treatment of the same presenting symptoms.

\section{Discussion}

Postprandial hypoglycemia, $\mathrm{HIH}$, is a complication seen in a subset of patients following GB surgery. It is an insidious complication that does not present immediately after surgery but evolves in patients who have progressively worsening dumping syndrome. This is suspected to be caused by GLP-1 stimulation and beta-cell hypertrophy. These patients may present with a broad spectrum of clinical findings, commonly with neuroglycopenia, diaphoresis, significant nausea, and weakness. Our experience indicates that the revision of gastric bypass to the duodenal switch resolves the HIH condition. Restoring normal pyloric function reverses dumping syndrome complications, eliminating postprandial hypoglycemia, and resolves the symptoms of Nesidioblastosis. Unlike near-total pancreatectomy, the duodenal switch procedure does not cause insulin insufficiency and reversed $100 \%$ of our patients' symptoms.

\section{Conclusion}

Continuous follow-up and observation of these fifteen patients have shown complete resolution of initial post-GB complications. Patients have reported a significant improvement in the quality of life. Risk versus benefit analysis in treating patients with reactive hypoglycemia and Nesidioblastosis with DS operation has shown more significant benefit than risk. In our opinion, the duodenal switch operation is a safer procedure to perform than a near-total pancreatectomy. It should be considered as the treatment of choice for a patient suffering the complication of reactive hypoglycemia and $\mathrm{Ne}$ sidioblastosis following a gastric bypass operation.

\section{Financial Disclosure}

None. 
Citation: Keshishian A, Rajtar M, Rosado M (2021) Surgical Treatment of Clinically Significant Reactive Hypoglycemia Nesidioblastosis, PostGastric Bypass. J Surgical Endocrinol 3(1):73-76

\section{References}

1. Cummings DE, Overduin J, Foster-Schubert KE (2004) Gastric bypass for obesity: Mechanisms of weight loss and diabetes resolution. J Clin Endocrinol Metab 89: 2608-2615.

2. Foster-Schubert KE, Cummings DE (2006) Emerging therapeutic strategies for obesity. Endocr Rev 27: 779-793.

3. Greenberger NJ, Robinson MK (2012) Treatment of obesity: The impact of bariatric surgery. In: Norton J. Greenberger, Richard S Blumberg, Robert Burakoff, Current diagnosis \& treatment: Gastroenterology, hepatology, \& endoscopy. ( $3^{\text {rd }}$ edn), The McGraw-Hill Companies, New York, N.Y.

4. Singh E, Vella A (2012) Hypoglycemia after gastric bypass surgery. Diabetes spectrum 25: 217-221.

5. Foster-Schubert KE (2011) Hypoglycemia complicating bariatric surgery: Incidence and mechanisms. Curr Opin Endocrinol Diabetes Obes 18: 129-133.

6. Anthone GJ, Lord RVN, DeMeester TR, et al. (2015) The duodenal switch operation for the treatment of morbid obesity. Ann Surg 238: 618-628.

7. DeMeester TR, Fuchs KH, Ball CS, et al. (1987) Experimental and clinical results with proximal end-to-end duodenojejunostomy for pathologic duodenogastric reflux. Ann Surg 206: 414-426.

8. Hess DS, Hess DW (1998) Biliopancreatic diversion with a duodenal switch. Obes Surg 8: 267-282.

9. (2007) Bariatric surgery: Postoperative concerns. American Society for Metabolic and Bariatric Surgery.

10. Sigstad H (1970) A clinical diagnostic index in the diagnosis of the dumping syndrome. Acta Med Scand 188: 479-486.

11. McLaughlin T, Peck M, Holst J, et al. (2010) Reversible hyperinsulilnemic hypoglycemia after gastric bypass: A consequence of altered nutrient delivery. J Clin Endocrinol Metab 95: 1851-1855.

12. Service GJ, Thompson GB, Service FJ, et al. (2005) Hyperinsulinemic hypoglycemia with nesidioblastosis after gastric-bypass surgery. N Engl J Med 353: 249-254.

13. Patti ME, McMahon G, Mun EC, et al. (2005) Severe hypoglycemia post-gastric bypass requiring partial pancreatectomy: Evidence for inappropriate insulin secretion and pancreatic islet hyperplasia. Diabetologia 48: 2236-2240.

14. Ukleja A (2005) Dumping syndrome: Pathophysiology and treatment. Nutr Clin Pract 20: 517-525.

15. Meier JJ, Butler AE, Galasso R, et al. (2006) Hyperinsulinemic hypoglycemia after gastric bypass surgery is not accompanied by islet hyperplasia or increased $\beta$-cell turnover. Diabetes Care 29: 1554-1559.

16. Scavini M, Pontiroli A, Folli F (2005) Asymptomatic hyperinsulinemic hypoglycemia after gastric banding. N Engl J Med 353: 2822-2823.

17. Xu G, Stoffers DA, Habener JF, et al. (1999) Exendin-4 stimmulates both beta-cell replication and neogenesis, resulting in increased beta-cell mass and improved glucose tolerance in diabetic rats. Diabetes 48: 2270-2276.

18. Farilla L, Hui H, Bertolotto C, et al. (2002) Glucagon-like peptide-1 promotes islet cell growth and inhibits apoptosis in Zucker diabetic rats. Endocrinology 143: 4397-4408.

19. Farilla L, Butolla A, Hirshberg B, et al. (2003) Glucagon-like peptide 1 inhibits cell apoptosis and improves glucose responsiveness of freshly isolated human islets. Endocrinology 144: 51495158.

20. Service FJ (1999) Classification of hypoglycemic disorders. Endocrinol Metab Clin North Am 28: 501-517.

21. Golfine AB, Mun EC, Devine E, et al. (2007) Patients with neuroglycopenia after gastric bypass surgery have exaggerated incretin and insulin secretory responses to a mixed meal. J Clin Endocrinol Metab 92: 4678-4685.

22. Lev-Ran A, Anderson RW (1981) The diagnosis of postprandial hypoglycemia. Diabetes 30: 996-999.

23. Kim SH, Liu TC, Abbasi F, et al. (2009) Plasma glucose and insulin regulation is abnormal following gastric bypass surgery with or without neuroglycopenia. Obes Surg 19: 1550-1556.

24. Manson JE, Skerrett PJ, Greenland P, et al. (2004) The escalating pandemics of obesity and sedentary lifestyle: A call to action for clinicians. Arch Intern Med 164: 249-258.

25. Tack J, Arts J, Caenepeel P, et al. (2009) Pathophysiology, diagnosis and management of postoperative dumping syndrome. Nat Rev Gastroenterol Hepatol 6: 583-590.

26. Hamilton L, Rohan Jeyarajah D (2007) Hemoglobin A1c can be helpful in predicting progression to diabetes after Whipple procedure. HPB (Oxford) 9: 26-28.

27. Ho CK, Kleeff J, Friess H, et al. (2005) Complications of pancreatic surgery. HPB (Oxford) 7: 99-108.

28. Fong T-L, Warner NE, Kumar D (1989) Pancreatic nesidioblastosis in adults. Diabetes Care 12: 108-114.

29. Peeters A, Barendregt JJ, Willekens F, et al. (2003) Obesity in adulthood and its consequences for life expectancy: A life-table analysis. Ann Intern Med 138: 24-32.

DOI: $10.36959 / 608 / 451$

Copyright: (C) 2021 Keshishian A, et al. This is an open-access article distributed under the terms of the Creative Commons Attribution License, which permits unrestricted use, distribution, and reproduction in any medium, provided the original author and source are credited. 